\title{
Alcune certezze e molti dubbi
}

\author{
Commento all'articolo: "Medical management to prevent recurrent nephrolithiasis in adults: \\ A systematic review for an American College of Physicians Clinical Guideline"
}

\author{
Alberto Trinchieri \\ S.C. Urologia, Ospedale “A. Manzoni”, Lecco
}

La recente metanalisi di Fink et al (1) descrive in modo estensivo le evidenze da studi controllati che hanno indagato l'efficacia di provvedimenti dietetici e farmacologici per la prevenzione della nefrolitiasi.

Una prima considerazione dai risultati della review, è la carenza di studi controllati di buona qualità che si prefiggano l'endpoint primario di ridurre il rischio di formazione di nuovi calcoli sintomatici o la comparsa o modificazione agli esami per immagini di calcoli in sede reno-ureterale. Questo outcome è molto solido ma implica un fattore di confondimento legato alle modalità di rilevazione e misurazione dei calcoli.

È stato possibile prendere in considerazione solo 30 studi controllati che sono stati classificati come "good" in solo 2 casi, mentre per la maggior parte degli studi il giudizio è stato "fair" e in 2 "poor". In solo 10 i risultati sono valutati secondo "intention to treat", sebbene la descrizione dei pazienti persi al follow-up sia presente in 23 e in 5 non vengano riportate defezioni durante il periodo di osservazione. Gran parte degli studi sono indirizzati alla prevenzione della calcolosi calcica, solo 2 alla calcolosi da infezione e nessuno alla calcolosi radiotrasparente. Le modalità di intervento sono molto eterogenee, sia per quanto riguarda gli studi basati su provvedimenti dietetici che per quelli farmacologici. Il panorama complessivo è abbastanza sconfortante, soprattutto se si tiene conto che gran parte degli studi è datato a più di 10 anni fa. I motivi di così scarsa produzione sono noti: ridotta motivazione dei pazienti che si limitano al trattamento non invasivo dei singoli episodi litiasici, elevato impegno organizzativo ed economico richiesto da questo tipo di indagini e, conseguentemente, scarso interesse dell'industria farmaceutica.

Un'analisi più specifica dei singoli provvedimenti terapeutici consente tuttavia di trarre alcune considerazioni.

L'efficacia della idropinoterapia, che è unanimemente riconosciuta dai clinici e dalle principali linee-guida, è suffragata da un unico studio controllato, con basso livello di qualità e limitato a pazienti formatori di un solo calcolo di qualsiasi composizione. Mancano informazioni sulla quantità e qualità dei liquidi che possono essere consigliati ai formatori di calcoli renali in relazione alla recidivanza e al tipo di calcolosi. Esiste solo una modesta evidenza, derivata da un'analisi post-hoc, che identifica come fattore protettivo la riduzione del consumo di bevande acidificate con acido fosforico in pazienti che in precedenza ne facevano un elevato consumo.

Il ruolo di altri possibili provvedimenti dietetici rimane di incerta valutazione, in quanto gli studi clinici randomizzati sono molto disomogenei in funzione del tipo e della durata degli interventi, del disegno e delle popolazioni studiate. In particolare si tratta di indicazioni dietetiche multiple intese a modificare contemporaneamente l'apporto di diversi nutrienti che rendono difficile valutare l'effetto di ogni singolo intervento. Inoltre la compliance è difficile da valutare, sebbene sia riferita come scarsa in molti studi e comunque inferiore a quella ottenuta negli studi di intervento farmacologico. Il tasso di dropouts raggiunge percentuali del 50-60\% in un periodo di 4 anni. Solo nello studio di Borghi la percentuale di dropouts è inferiore al $15 \%$ in un periodo di 5 anni. D'altra parte anche nei pazienti che si presentano regolarmente ai controlli è difficile identificare coloro che adeguano correttamente la loro dieta al protocollo di studio. La compliance potrebbe essere basata su autovalutazione (recalls, questionari) o sulla valutazione di markers urinari (sodio, solfati, urea) che non sono sempre previsti nei protocolli sperimentali. Infine deve essere rilevato che quasi tutti gli studi di intervento dietetico sono stati indirizzati a pazienti dopo il primo episodio litiasico e non a quelli recidivanti, il che implica lunghi periodi di osservazione affinché si concretizzi il rischio litiasico.

La metanalisi degli studi farmacologici è più soddisfacente in quanto l'impiego dei gruppi farmacologici principali (tiazidici, citrati, allopurinolo) dimostra adeguati livelli di efficacia nonostante siano state impiegate diverse molecole della classe dei tiazidici e diversi sali di citrato.

I dati disponibili dimostrano l'efficacia della terapia farmacologica in generale, mentre mancano evidenze relative alla possibilità di poter eseguire un trattamento "mirato" alla correzione di specifici difetti metabolici. L'allopurinolo si dimostra efficace in pazienti con alterazioni del metabolismo dell'acido urico, tuttavia non può essere considerato l'unico farmaco efficace in questi pazienti. La compliance al trattamento, in questi studi randomizzati, è superiore rispetto a quella osservata negli studi di intervento dietetico, ma questo risultato potrebbe essere spiegato dal fatto che il trattamento farmacologico è stato proposto in pazienti selezionati per la maggior recidivanza, quindi potenzialmente più motivati al trattamento.

In conclusione, gran parte dei provvedimenti dietetici che abitualmente vengono consigliati ai pazienti con calcolosi 
non trova solide basi di evidenza in studi controllati, sebbene esistano evidenze dedotte da studi osservazionali di coorte numericamente consistenti. Viceversa esiste una discreta evidenza dell'efficacia dei provvedimenti farmacologici, tuttavia non sufficiente per dimostrare la possibilità di una terapia mirata alla correzione di singoli difetti del metabolismo.

Dichiarazione di conflitto di interesse: L'Autore dichiara di non avere conflitto di interessi.

Contributi economici agli Autori: L'Autore dichiara di non avere ricevuto sponsorizzazioni economiche per la preparazione dell'articolo.

Indirizzo dell'Autore:

Dr. Alberto Trinchieri

S.C. Urologia

Ospedale "A. Manzoni"

Via dell'Eremo 9/11

23900 Lecco

a.trinchieri@ospedale.lecco.it

\section{Bibliografia}

1. Fink HA, Wilt TJ, Eidman KE, et al. Medical management to prevent recurrent nephrolithiasis in adults: a systematic review for an American College of Physicians Clinical Guideline. Ann Intern Med 2013; 158(7): 535-43. 